Revue pluridisciplinaire d'études médiévales

\title{
Diffusion et érosion du culte de saint Michel dans le sud du Grésivaudan (Dauphiné, $\mathrm{XI}^{\mathrm{e}}-\mathrm{XVII}{ }^{\mathrm{e}}$ siècles)
}

\section{Bruno Varennes}

\section{OpenEdition}

Journals

Édition électronique

URL : http://journals.openedition.org/questes/1079

DOI : 10.4000/questes. 1079

ISSN : 2109-9472

\section{Éditeur}

Les Amis de Questes

\section{Édition imprimée}

Date de publication : 15 septembre 2010

Pagination : 23-36

ISSN : 2102-7188

\section{Référence électronique}

Bruno Varennes, « Diffusion et érosion du culte de saint Michel dans le sud du Grésivaudan (Dauphiné, xI ${ }^{e}$-xvII ${ }^{e}$ siècles) », Questes [En ligne], 19 | 2010, mis en ligne le 01 janvier 2014, consulté le 18 septembre 2020. URL : http://journals.openedition.org/questes/1079 ; DOI : https://doi.org/10.4000/ questes. 1079 


\title{
Diffusion et érosion du culte de saint Michel dans le sud du Grésivaudan (Dauphiné, $\mathrm{XI}^{\mathrm{e}}-\mathrm{XVII}{ }^{\mathrm{e}}$ siècles)
}

\author{
Bruno VARENNES ${ }^{1}$
}

$\mathrm{Au}$ sein des apparitions divines, celles des saints intercesseurs occupent une place particulière. L'archange saint Michel est un exemple singulier, chacune de ses interventions terrestres ayant marqué un terroir par la mise en place d'un sanctuaire. L'étude de la diffusion du vocable - le nom du saint auquel une église doit son appellation - permettra de connaître l'écho qu'a eu son culte à l'intérieur d'une province traversée par les voies de communication raccordant le centre de la France à Rome, mettant ainsi en relations les principaux sanctuaires michaéliques. Il s'agira de comprendre le rôle joué par l'archange au cœur du Moyen Âge malgré l'absence de manifestations connues, avant de s'intéresser à la désaffection de son culte.

Le 8 mai 490 eut lieu sa première apparition en Occident au Monte Gargano, terre hellénisée dans le nord des Pouilles. Saint Michel ordonna à l'évêque Lorenzo Maiorano de lui consacrer un lieu de culte avant de réapparaître en 492 et 493, réaffirmant son intérêt pour le site qu'il marqua de l'empreinte de son pied au «IX $\mathrm{IX}^{\mathrm{e}}$ siècle seulement $»^{2}$. En 708-709, il choisit le Monte que dicitur tomba (Mont Saint-Michel) pour se manifester, ordonnant à l'évêque d'Avranches Aubert l'érection d'un oratoire à l'exemple du Gargano. Enfin, à la veille de l'an mille se développe un

\footnotetext{
1 Doctorant Université de Grenoble, thèse en cours intitulée $D u$ lieu de culte à l'Ecclesia. Espaces, Réseaux, Édifices à la croisée des diocèses de Die et de Grenoble $\left(X I I^{e}-X V I^{e}\right.$ siècles), sous la direction de Dominique Rigaux. Une partie des conclusions présentées ici (figures 2, 3 et 4) sont issues de ces recherches.

${ }^{2}$ Anne Wagner, Les Saints et l'histoire : sources hagiographiques du haut Moyen Âge, Paris, Bréal, 2004, p. 8.
} 
sanctuaire sur le Monte Pirchiriano (La Sacra di San Michele), à l'entrée du Val di Susa ${ }^{3}$. C'est sur cet « axe majeur », construit depuis le Gargano et traversant l'Europe occidentale via la Sacra di San Michele jusqu'au Mont Saint-Michel, que se développa le culte au $\mathrm{X}^{\mathrm{e}}$ siècle (figure 1$)^{4}$.

\section{Figure 1 - Carte. Les principaux sanctuaires michaéliens d'Occident}

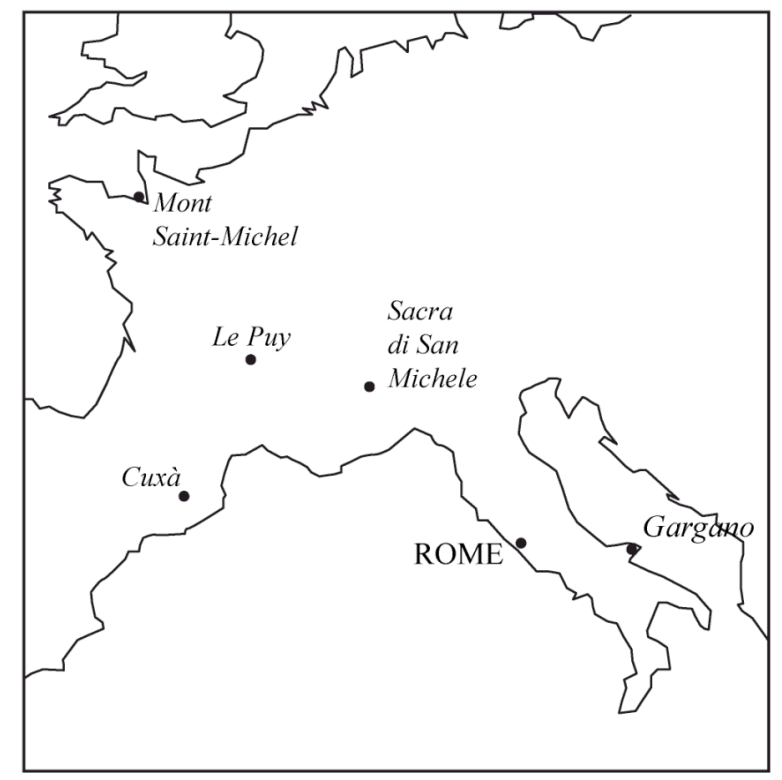

${ }^{3} \mathrm{Sa}$ fondation remonte aux « années 983-987, par l'ermite Jean Vincent (...) et le chevalier auvergnat Hugues le Décousu ». Véronique GAZEAU et Monique Goullet, Guillaume de Volpiano, un réformateur en son temps (962-1031): Vita domni Wilhelmi de Raoul Glaber. Texte, traduction, commentaire, Caen, Université de BasseNormandie, Publications du CRAHM, 2008, p. 92.

4 «Une basilique lui fut construite à Rome dès le $\mathrm{V}^{\mathrm{e}}$ siècle, suivie d'une chapelle édifiée par Boniface IV au château Saint-Ange ». Cependant, pour Michel Aubrun, "davantage que les Italiens, ce furent donc les Normands, puis surtout les Irlandais qui répandirent le culte rendu à ce personnage céleste ». Christian Lauranson-Rosaz rappelle que la consécration « au Puy de l'oratoire fondé (...) en 962 au sommet du piton volcanique d'Aiguilhe, a lieu solennellement en 993. Les fondations des trois autres grands oratoires occidentaux (...) ont lieu dans un intervalle qui n'excède pas vingt ans : Le Mont-Tombe en 961, Cuxa entre 956 et 974, La Cluse entre 966 et 983/7 ». Michel Aubrun, L'ancien diocèse de Limoges des origines au milieu du XI siècle, ClermontFerrand, Institut d'étude du Massif Central, 21, 1981, p. 332. Christian LAURANSONRosAz, « De la chiusa à Cuixà. La Romania de l'an Mil sous le signe de l'archange Michel et de Saint Pierre ", in L'An Mil. Fin d'un monde ou renouveau? Les Cahiers de Saint-Michel de Cuxa, Codalet XXXII (2001), p.98. Voir aussi Giampietro Casiraghi, Giuseppe Sergi (a cura di), Pellegrinaggi e santuari di San Michele nell'Occidente medievale, Bari, Edipuglia (Bibliotheca michaelica, vol. 5), 2009. 
Les sanctuaires sont le « lieu de médiation privilégié entre le sacré et le profane ». À ce titre, ils revêtent « un rôle particulier dans les relations entre un groupe humain et le surnaturel $»^{5}$. Cette fonction est liée aux attributs psychopompes du saint et à la position des sanctuaires qui lui sont consacrés essentiellement sur les sommets, en référence au Gargano ${ }^{6}$. Michel est d'abord un guerrier, mais il est également un gardien dans la vie et un protecteur lors du passage vers l'au-delà, au moment de la pesée des âmes, ce que confirme une étude iconographique récente sur ses représentations dans les Alpes ${ }^{7}$. Sa titulature pour un autel majeur, un simple oratoire ou la dédicace d'une communauté paroissiale, révèle la recherche de cette double protection du saint. De ce fait, ces sanctuaires ont joué un rôle important dans la diffusion du culte.

\section{Diffusion et implantation du culte dans une région du Dauphiné alpin}

Les sites d'implantation du culte dans la montagne dauphinoise permettent d'appréhender le phénomène de diffusion sur un territoire donné.

\footnotetext{
5 Monica SARACCO, «Il culto di san Michele nell'Italia settentrionale sondaggi e prospettive d'indagine ", in Pierre Bouet, Giorgio Otranto, André VAuChEZ (a cura di), Culto e santuari di san Michele nell'Europa medievale, Bari, Edipuglia (Bibliotheca michaelica, vol. 1), 2007, p. 219.

${ }^{6}$ Cette prédilection pour les cimes n'a pas limité le développement du culte sur d'autres espaces. Dans les îles britanniques, Graham Jones distingue les établissements de plaine, caractérisés par la présence de l'eau, rappel du baptême, et ceux des hauteurs se réclamant de l'exemple du Gargano. Graham Jones, "The cult of Michael the archangel in Britain », in Pierre Bouet, Giorgio OtRanto, André VAuchez, (a cura di), Culto e santuari di san Michele nell'Europa medievale, op. cit., p. 147-182. Voir aussi Giorgio OTRANTO, « Note sulla tipologia degli insediamenti micaelici nell'Europa medievale », ibid., p. 385-418.

7 Dominique RigauX, «Michel et l'ultime voyage. Iconographie médiévale de l'archange dans les vallées alpines », in Giampietro CASIRAGHI, Giuseppe SERGI (a cura di), Pellegrinaggi e santuari di San Michele nell'Occidente medievale, op. cit., p. 577597.
} 
Les routes en direction de Rome permettent la diffusion du culte ${ }^{8}$. Or, l'apparition du Pirchiriano (Sacra di San Michele) a eu lieu sur la voie reliant le Massif Central et Rome, amenant le pèlerinage qui entretient le sanctuaire ${ }^{9}$. Le Dauphiné est à la croisée de chemins menant vers Rome et reliant les différents sanctuaires: une capella Sancti Michaelis de Grationopoli (Grenoble) est signalée dès $1109^{10}$.

À l'intérieur de la province, notre enquête porte sur un territoire réduit aux confins des diocèses de Die et de Grenoble, sur la route menant de cette dernière à Aix-en-Provence. Marquée par des influences auvergnates, provençales, et encore cisalpines, la région a été le théâtre d'une « reconquête » chrétienne avant et autour de l'an mille, dans laquelle le rôle de l'archange ne semble pas neutre. Le couloir formé par le revers oriental du Vercors, espace de contact entre les diocèses de Die et de Grenoble, est le lieu d'implantation de nombreux lieux de culte qui lui sont dédiés. L'étude de cet espace nous permettra d'appréhender la diffusion du saint dans une zone alpine.

Dans cette partie du sud du Grésivaudan, le relief a contraint les implantations humaines ${ }^{11}$. Si les lieux du culte michaélique sont nombreux, les titulatures de chapelles sont secondaires, et les sites d'altitude dominent. L'archange apparait bien comme un saint des hauteurs, mais surtout vénéré par toutes les strates de la société.

\footnotetext{
${ }^{8}$ Ibid., p. 597.

${ }^{9}$ Pour l'attractivité du sanctuaire, signalons le passage de Guillaume de Volpiano « vers 987 ». Véronique GAZEAU et Monique Goullet, Guillaume de Volpiano, un réformateur en son temps, op. cit., p. 92.

${ }^{10}$ Jules MARIOn, Cartulaire de l'Église cathédrale de Grenoble, dits Cartulaires Saint Hugues, Paris, Imprimerie impériale, 1869, Cart. B, « Charte de Bonus Aur », 18 février 1109 , p. 98.

${ }^{11} \mathrm{Au} \mathrm{XI} \mathrm{e}^{\mathrm{e}}$ siècle, la «multiplicité des seigneuries comme des lieux d'habitat est (...) le reflet d'un relief et d'un réseau hydrographique tous deux causes de morcellement et d'enclavement », Chantal MAZARD, « Le Moyen Âge », in Patrimoine en Isère, Trièves, Grenoble, Conservation du patrimoine de l'Isère, 1996, p. 57.
} 
Figure 2 - Tableau. Les dédicaces à saint Michel

\begin{tabular}{|c|c|c|c|c|c|c|c|c|c|c|c|c|c|c|}
\hline 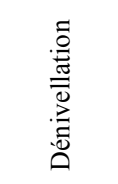 & 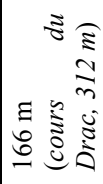 & 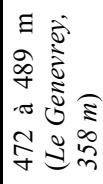 & 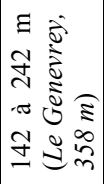 & 离 & \multicolumn{4}{|c|}{ 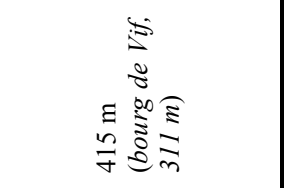 } & 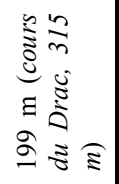 & 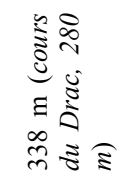 & 离 & \multicolumn{3}{|c|}{ 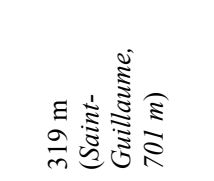 } \\
\hline Altitude & $\begin{array}{l}\Xi \\
\infty \\
\stackrel{\infty}{f}\end{array}$ & 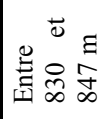 & 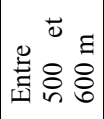 & $\stackrel{\Xi}{\circ}$ & \multicolumn{4}{|c|}{$\begin{array}{l}\mathbb{\Xi} \\
\stackrel{\sim}{N} \\
\text { N }\end{array}$} & $\frac{\Xi}{\dot{n}}$ & $\begin{array}{l}\Xi \\
\frac{\infty}{\sigma}\end{array}$ & $\begin{array}{l}\Xi \\
\stackrel{D}{\Xi} \\
=\end{array}$ & \multicolumn{3}{|c|}{$\begin{array}{l}\Xi \\
\stackrel{\Xi}{9} \\
\stackrel{\varrho}{a}\end{array}$} \\
\hline 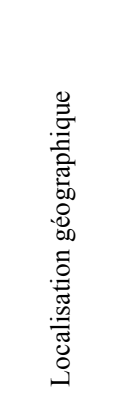 & 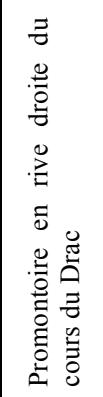 & 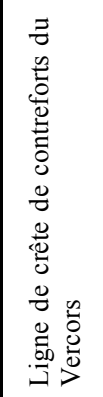 & 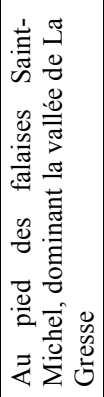 & 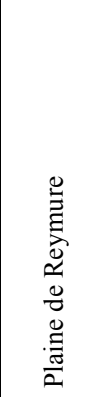 & \multicolumn{4}{|c|}{ 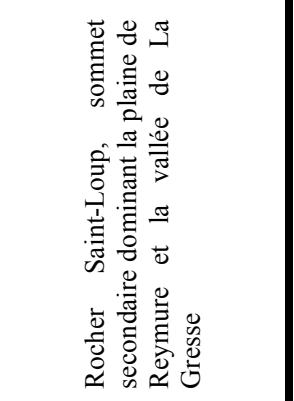 } & 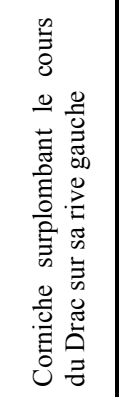 & 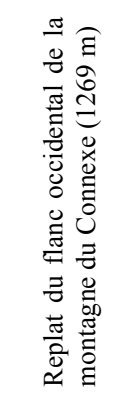 & 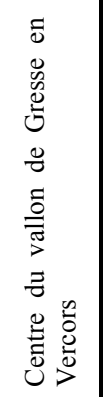 & \multicolumn{3}{|c|}{ 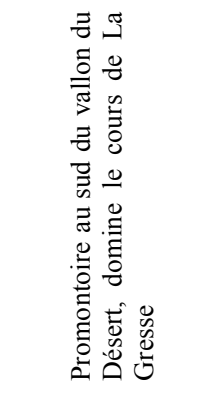 } \\
\hline \multirow{6}{*}{ 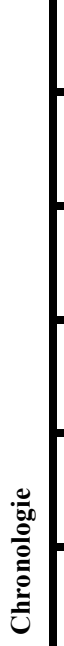 } & ' & & & & & & & & & , & $\stackrel{m}{6}$ & $\leftrightarrow$ & $\stackrel{m}{\sigma}$ & 苦 \\
\hline & ' & & & & & 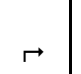 & : & $\stackrel{\bar{n}}{n}$ & , & , & $i$ & , & & \\
\hline & $\stackrel{\text { }}{ \pm}$ & & $T$ & $\stackrel{\substack{\infty \\
\pm}}{ \pm}$ & 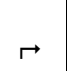 & $\sum_{\dot{x}}$ & & & $\begin{array}{l}\infty \\
\stackrel{\infty}{d} \\
d\end{array}$ & , & i & , & & \\
\hline & ' & & & & ले & & & & & ' & & . & & \\
\hline & . & 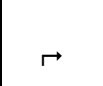 & $\stackrel{n}{\beth}$ & & & & & & & ' & & 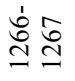 & & \\
\hline & , & 气ે & & & & & & & & $\stackrel{\varrho}{ٍ}$ & & & & \\
\hline $\begin{array}{l}\text { Dédicace } \\
\text { associée }\end{array}$ & , & & 言 & : & & 胥 & & : & & & & 节离 & & 震离 \\
\hline 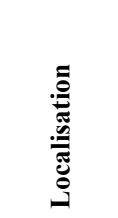 & 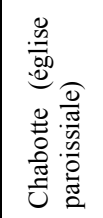 & 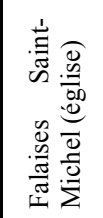 & 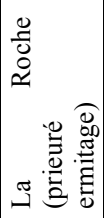 & 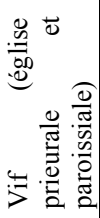 & & $\frac{0}{0}$ & 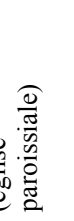 & & 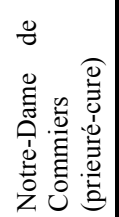 & 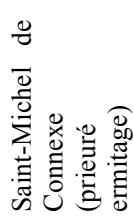 & 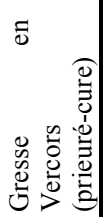 & \multicolumn{3}{|c|}{ 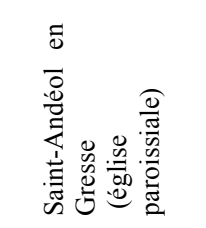 } \\
\hline \multirow[t]{2}{*}{ 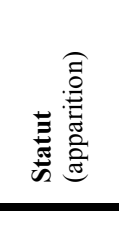 } & 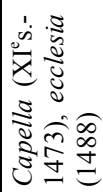 & 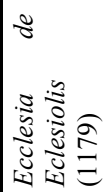 & 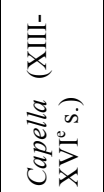 & 离 & & 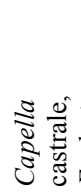 & 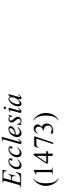 & & 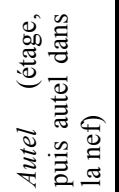 & 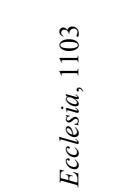 & 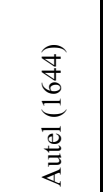 & & 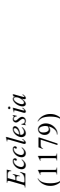 & \\
\hline & \multicolumn{10}{|c|}{ DIOCESE DE GRENOBLE } & \multicolumn{4}{|c|}{ DIOCESE DE DIE } \\
\hline
\end{tabular}


Figure 3 - Carte. Localisation des dédicaces à saint Michel aux confins des deux diocèses

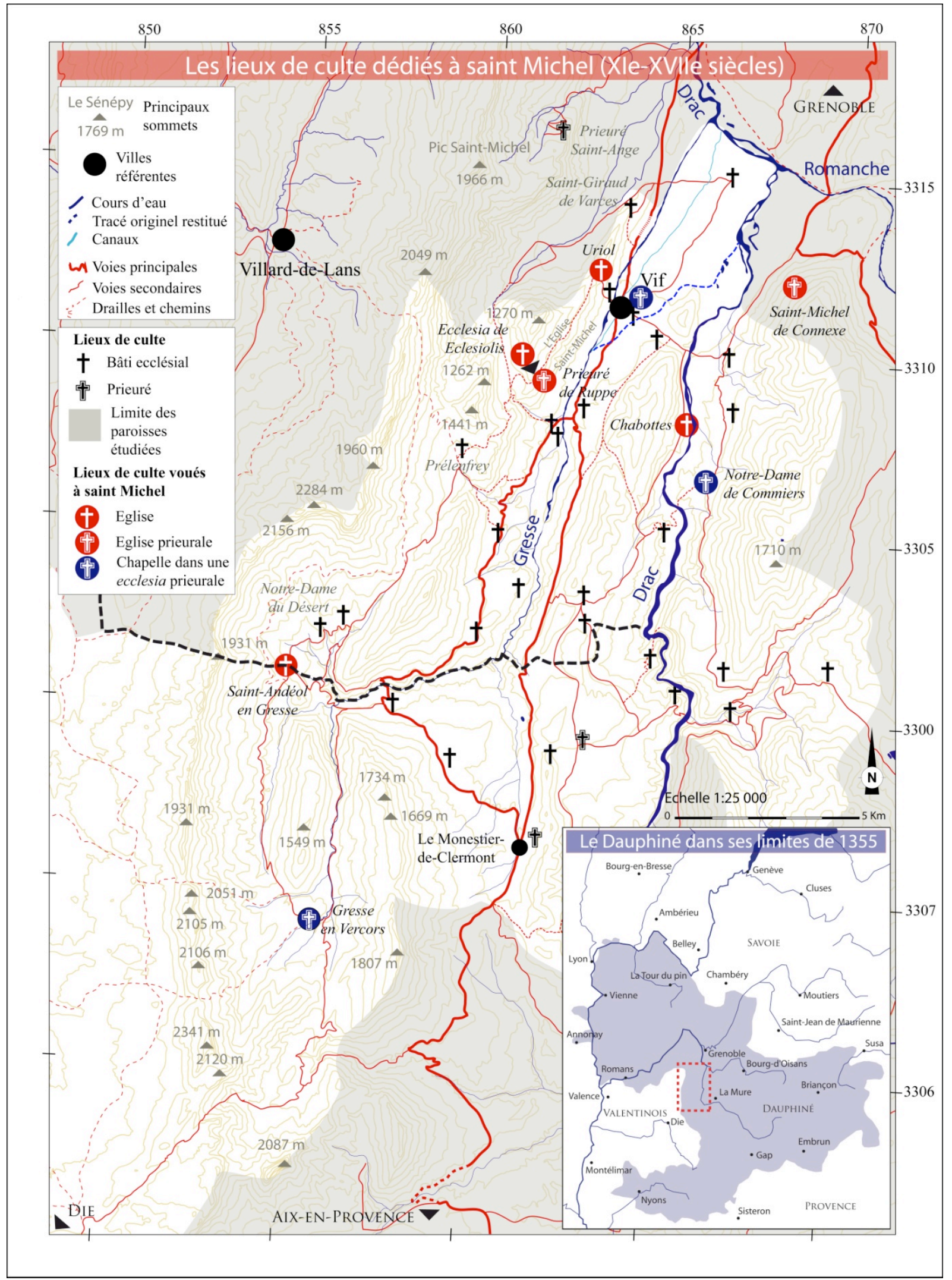


Le corpus démontre que les dédicaces au saint sont réservées à l'autel majeur dans les édifices construits en hauteur (figures 2 et 3). Depuis les cimes des contreforts au sud de Varces jusqu'à Gresse en Vercors, un bâti ecclésial sur deux a pris le saint comme titulature principale ou pour celle d'une chapelle secondaire entre le $\mathrm{XII}^{\mathrm{e}}$ et le XVII ${ }^{\mathrm{e}}$ siècle. Ainsi, du nord au sud, il est absent à Saint-Giraud de Varces alors qu'il est le premier vocable à Uriol (1339) où saint Loup le rejoint dans un second temps (XV $\mathrm{X}^{\mathrm{e}} \mathrm{s}$.). On le retrouve à Eclesiolis, ecclesia mentionnée en 1179, où il reste associé à saint Blaise, en dépit du transfert du lieu de culte au hameau de Ruppe lorsque s'implante un prieuré avant $1275^{12}$. Il n'apparaît pas dans les vallons de Prélenfrey et de Notre-Dame du Désert, mais sa titulature est révélée tardivement (1613) dans celle de Saint-Andéol (autel majeur) et comme dédicace d'une chapelle de Saint-Barthélemy de Gresse-enVercors. En contrebas, dans la basse vallée de La Gresse comme dans celle du Drac, son influence est moins forte. Seule l'ecclesia de Chabotte lui est consacrée, ainsi que deux chapelles, dressées dans les églises de SaintJean-Baptiste de Vif et de Notre-Dame de Commiers. Enfin, outre Drac, l'archange est le titulaire de l'autel majeur du prieuré de Connexe dont un prieur est connu dès $1103^{13}$.

Même si des sanctuaires existent dans les vallées, l'essentiel est en hauteur (figure 3). Notons que le vocable de saint Michel est l'un des rares

\footnotetext{
${ }^{12}$ Acte $\mathrm{n}^{\circ} 442$, Bulle de confirmation de possession par du pape Alexandre III, le $1^{\mathrm{er}}$ avril 1179, dans Jules MARION, Cartulaire de l'Église cathédrale de Grenoble, op. cit., p. 178-183 ; Comptes de Décimes de 1275 du diocèse de Grenoble, publiés par Joseph Joseph Calmette, Étienne Clouzot, Pouillé des provinces de Besançon de tarentaise et de Vienne, Paris, Imprimerie Nationale éd., 1940, p. 380.

${ }^{13}$ Gérard CARDIN, "Le prieuré chaffrien de Saint-Michel-de-Connexe (Isère) ", in Les Bénédictins de Saint Chaffre du Monastier : histoire et archéologie d'une congrégation, Actes du colloque des 7-9 novembre 1997, Le-Monastier-sur-Gazeille, 1998, p. 273.
} 
à avoir marqué la toponymie du Vercors ${ }^{14}$. C'est un sommet portant son nom qui borne le nord-est du massif $(1966 \mathrm{~m})$ où la disparition de l'ecclesia de Eclesiolis a laissé son empreinte : Les Falaises Saint-Michel. Cette localisation présente une recherche de mimétisme avec les sites d'implantations des principaux sanctuaires, et le Gargano en premier lieu.

Les autels secondaires sont insérés dans des prieurés-cures pour lesquels le relief ne joue aucun rôle. Ils sont le réceptacle de la piété locale à la fin du Moyen Âge. À Gresse, l'église rayonne sur un plateau d'altitude vallonné, alors qu'à Notre-Dame de Commiers la chapelle est superposée au chœur avant d'être transférée dans la nef au cours du $X V^{\mathrm{e}}$ siècle. Enfin, à Vif, elle s'insère dans un bâti ecclésial fortement remanié au XIII ${ }^{\mathrm{e}}$ siècle par le prieuré bénédictin attenant.

Si l'archange a marqué des édifices ruraux implantés loin des routes principales (Uriol, Saint-Andéol), il n'est pas l'apanage d'une catégorie de la population, mais un saint ouvert à tous. Outre les lieux de culte ruraux, il a été choisi pour un édifice castral (Uriol), et comme le saint tutélaire de deux prieurés ermitages (La Roche, Connexe). L'archange transcende les clivages ${ }^{15}$.

\footnotetext{
${ }^{14}$ D'autres cas existent, mais restent uniques : saint Loup a donné son nom au site d'Uriol, alors que l'appellation « la Côte Saint-Blaise » est conservée là où s'élevait le prieuré des saints Michel et Blaise.

${ }^{15}$ On rejoint ici le constat dressé par Dominique Rigaux : «L'iconographie alpine permet de mesurer le succès de la dévotion à l'archange en milieu rural et montagnard. Michel n'est pas seulement le saint de l'aristocratie, le guerrier invincible qui terrasse les forces du mal, il est aussi l'ange familier qui assiste les défunts et a le pouvoir de soustraire les âmes des justes à l'emprise du diable et de les conduire à la paix et au Paradis », Dominique RIGAuX, « Michel et l'ultime voyage. Iconographie médiévale de l'archange dans les vallées alpines », art. cit., p. 596-597.
} 


\section{Le culte : origine et déclin}

Le culte à saint Michel se répand tôt, mais évolue au fil des siècles, offrant des réponses en fonction des besoins changeants de la société. Ainsi, il apparaît comme une réaction aux tensions des $\mathrm{IX}^{\mathrm{e}}-\mathrm{X}^{\mathrm{e}}$ siècles puis, aux siècles suivants, se développe dans une religiosité plus personnelle. En dépit d'un culte basé à l'origine sur un phénomène d'apparition, aucun document ne dévoile de manifestation du saint ni d'un transfert de ses reliques dans la région. Il semble que l'on a surtout cherché à recréer les conditions de la venue de l'archange, comme si elles étaient suffisantes pour permettre son intercession.

En parallèle d'une propagation depuis la Sacra di San Michele, c'est la «re-christianisation » - à moins qu'il faille ici parler d'une véritable christianisation - du territoire montagnard et le développement d'une élite aristocratique et militaire qui semblent être à l'origine de l'implantation du vocable. Les plus anciennes mentions le présentent titulaire d'édifices ecclésiaux dont l'un est castral (Uriol) ${ }^{16}$. À Saint-Andéol, la double consécration mentionnée au $\mathrm{XVII}^{\mathrm{e}}$ siècle (figure 2) révèle un ancrage ancien, certainement antérieur à la Réforme Grégorienne qui amena la seconde dédicace ${ }^{17}$.

Nous l'avons déjà évoqué, saint Michel apparaît comme un saint guerrier. «C'est à ce titre qu'il est le défenseur de l'Église et le patron de plusieurs ordres de chevalerie (...), et de presque tous les corps de métiers liés aux armes ${ }^{18}$. Il est aussi l'un des canalisateurs de la force naissante de

\footnotetext{
${ }^{16}$ Yves Armand, Jean-Claude Michel, Histoire de Vif, Vif, Mairie de Vif éd., 2006, p. 64.

${ }^{17}$ La seconde dédicace, plus méridionale, est liée à la structuration des frontières diocésaines et rattache l'édifice au diocèse de Die. Pour une vie de saint Andéol, voir P. BurCHIL, "Santo Andeolo », Bibliotheca Sanctorum, Istituto Giovanni XXIII della Pontificia Università Laternense, vol. VIII, Roma, 1967, vol. 1, col. 1091-1092.

18 Dominique RigauX, «Michel et l'ultime voyage. Iconographie médiévale de l'archange dans les vallées alpines », art. cit., p. 577.
} 
l'aristocratie guerrière, pour laquelle l'Église met en avant l'exemple des milites christi. La présence de sites consacrés à «saint Michel, comme saint Pierre, tous deux gardiens et protecteurs des enceintes sacrées que sont le Paradis céleste ou les parvis d'églises » démontre un rôle pacificateur $^{19}$. «Le développement flagrant de leurs cultes dans la seconde moitié du $\mathrm{X}^{\mathrm{e}}$ siècle est une réponse évidente à la crise que connaît alors la société méridionale, la trop fameuse "mutation féodale" ", ce qui est ici aussi le $\operatorname{cas}^{20}$. L'archange n'est pas le seul saint guerrier présent. Outre Pierre, titulaire de l'église de Clermont, les communautés voisines offrent des patronages proches, Géraud dans le castrum éponyme de Varces et Maurice à Fontanieu.

La présence de ces saints guerriers doit être mise en relation avec un phénomène particulier. En effet, c'est une période confuse de brigandage liée à la présence endémique des sarrasins et des cavaliers hongrois dans le sud de l'arc alpin qui précède le temps de la Réforme Grégorienne. Au diocèse de Grenoble, l'évêque Isarn (v. 949 - v. 990) participa à l'éviction des derniers brigands «marrons » des Alpes ${ }^{21}$. En fait, l'insécurité due aux raids magyars est secondaire. La présence sarrasine reste «difficile à apprécier », mais la collusion avec les populations locales l'atteste ${ }^{22}$. Ce sont des «bandits plus ou moins rassemblés sous la bannière sarrasine,

${ }^{19}$ Christian Lauranson-Rosaz, « De la chiusa à Cuixà. La Romania de l'an Mil sous le signe de l'archange Michel et de Saint Pierre », art. cit., p. 99

${ }^{20}$ Idem.

${ }^{21}$ Les principaux textes de ce dossier ont été rédigés par les clercs de l'Église cathédrale de Grenoble, alors que l'évêque Hugues de Châteauneuf se bat pour la libertas ecclésiastique (1080-1132) face à l'archevêque de Vienne. Cette source n'est assurément pas objective puisqu'elle doit légitimer les droits de l'épiscopat en pleine Réforme Grégorienne. Henri FALQUe-Vert, Les Paysans et la terre en Dauphiné vers l'an Mil, Grenoble, Presses Universitaires de Grenoble, coll. "La Pierre et l'Écrit», 2004, p. 25. Voir aussi Laurent RIPART, « Du comitatus à l'episcopatus. Le partage du pagus de Sermorens entre les diocèses de Vienne et de Grenoble (1107) », in Florian MAZEL (dir.), L'Espace du diocèse. Genèse d'un territoire dans l'Occident médiéval ( $V^{e}$-XIII siècle), Rennes, Presses Universitaires de Rennes, 2008, p. 253-286.

${ }^{22}$ René FAVIER (dir.), Nouvelle histoire du Dauphiné, Grenoble, Glénat, 2007, p. 37. 
mais aux origines au moins autant régionales que méditerranéennes, et dont en tout cas l'identité religieuse n'est ni cohérente ni essentielle ». Eudes de Cluny parle de "Marrons, ces cruels habitants des Alpes (ipse quippe Marruci rigentes videlicet Alpium incolae) qui n'estimaient rien de plus avantageux que de transporter les bagages de Géraud» d'Aurillac. "Guides dans la traversée des Alpes, autant que brigands », ce sont des autochtones qui «ont pactisé avec les bandes de pillards sarrasins » ${ }^{23}$. Ils forment un danger réel : « entre 921 et 942 , on a pu dénombrer pas moins de dix expéditions dans les vallées alpestres », lors desquelles les grandes abbayes sont détruites (La Novalaise, la collégiale d'Oulx, toutes deux sur les routes de cols importants). Les pèlerins qui traversent les Alpes pour rejoindre Rome le font à leurs risques et périls, tel l'évêque de Tours en 931 puis l'abbé de Cluny Mayeul en $972-973^{24}$.

Le besoin de protection est souligné dans les fondations monastiques : les prieurés ermitages du territoire ont choisi cette dédicace. De plus, le prieuré Saint-Michel de Connexe s'est doté d'une dépendance entre la fin du XIII ${ }^{\mathrm{e}}$ siècle et le début du siècle suivant. Si la chapelle de

${ }^{23}$ « Il n'est pas d'autres moyens de comprendre que des étrangers, à l'origine des marins, aient pu, pendant près d'un siècle, fréquenter et surtout contrôler les petits chemins et sentiers qui permettaient alors la traversée des Alpes. Leurs guides et leurs alliés furent des "chrétiens" (est-ce sûr ?) insoumis, ceux que Raoul le Glabre, quelques décennies plus tard et après la disparition des sarrasins, traitera encore de "brutes gentes" et de "peuplades stupides" ", Henri FALQue-Vert, Les Paysans et la terre en Dauphiné vers l'an Mil, op. cit., p. 23-25.

${ }^{24}$ En l'an 931 se produit l'assassinat de l'évêque de Tours et de ses compagnons «par des pillards (latronibus) », « au pied des Alpes (sub alpibus)». Eudes abbé de Cluny [dans Vie de saint Géraud d'Aurillac, rédaction au second quart du $\mathrm{X}^{\mathrm{e}}$ siècle, vers 927 942] "rapporte le vol de bêtes de sommes dérobées à ce puissant pèlerin, les attaques de brigands, et précise "depuis longtemps déjà, les sarrasins ravageaient cette région", c'est-à-dire "la route qui de la cité de Turin gagne Lyon", sans doute par le col du MontCenis et la vallée de 1'Arc », ibid., p. 23-25. Voir aussi Monique ZERNER, « La capture de Mayeul et la guerre de libération en Provence : le départ des Sarrasins vu à travers les cartulaires provençaux ", in Saint Mayeul et son temps, Digne, Société scientifique et littéraire des Alpes de Haute-Provence, 1997, p. 199-210. 
cette cella, érigée au pied du Mont-Saint-Michel du Vercors, est vouée à saint Imbert, ce petit prieuré est dédié à saint Ange ${ }^{25}$.

La dédicace d'un lieu de culte communautaire atteste bien de l'intercession du saint. Cette intercession est encore plus visible lors de la fondation d'un autel secondaire. Titulaire de chapelles disposées à l'intérieur d'édifices cultuels au rayonnement important, l'archange participe à l'éclosion de chapellenies de la fin du Moyen Âge, bien visible en Dauphiné ${ }^{26}$. Son autel n'est jamais isolé dans les nefs, il est même dans certain cas associé à un autre saint, comme à Vif (figure 2). Lors de la reconstruction du chœur de Notre-Dame de Commiers, la chapelle est implantée au-dessus du sanctuaire : ce schéma se rapproche du modèle monastique d'une chapelle haute dans une tour, car l'élévation du chœur fait office de clocher ${ }^{27}$. Ainsi, bien que les chapelles participent d'un culte plus intériorisé, elles forment surtout un lien plus direct avec l'au-delà. À Vif, l'autel connu à partir de la fin du $X^{\mathrm{e}}$ siècle est situé dans le bas-côté nord de la nef, où il fait le lien avec le cimetière implanté au-delà du mur gouttereau. Il est en contrebas d'un décor peint représentant le réveil des

${ }^{25}$ Un compte de taxe mentionne un prieur dès 1275, alors qu'Emmanuel Pilot de Thorey ne cite pas de lieu de culte avant 1307. Joseph CALMETTE, Étienne Clouzot, Pouillé des provinces de Besançon de tarentaise et de Vienne, op. cit., p. 380-389. Emmanuel PILOT DE THOREY, «Les prieurés de l'ancien diocèse de Grenoble compris dans les limites du Dauphiné », Bulletin de la Société de Statistique des Sciences Naturelles et des Arts Industriels du Département de l'Isère, $3^{\mathrm{e}}$ série, tome XII, XXIII ${ }^{\mathrm{e}}$ de la collection (1883), p. 249-250.

${ }^{26}$ Voir à ce sujet Pierrette PARAVY, De la Chrétienté Romaine à la Réforme en Dauphiné, Rome, École Française de Rome, 1993.

${ }^{27}$ On rappellera à ce titre l'exemple signalé par les écrits de Pierre Le Vénérable qui expose, dans son De Miraculis, l'histoire d'un frère dénommé Benoît " saint homme » qui « avait pour cellule un oratoire consacré à l'archange saint Michel, dans une tour très haute et très à l'écart ». Pierre Le Venerable, Les Merveilles de Dieu (De Miraculis), présenté par Jean-Pierre TORREL, Denise BouTHILliER, Fribourg - Paris, Éditions Universitaires de Fribourg, 1992, p. 140 et 143. Christian Lauranson-Rosaz rappelle qu' " à l'époque romane, saint Michel, auquel sont dédiées nombre de chapelles hautes placées à l'ouest des sanctuaires, est considéré comme le saint militaire défenseur des portes ». Christian LaURAnson-Rosaz, " De la chiusa à Cuixà. La Romania de l'an Mil sous le signe de l'archange Michel et de Saint Pierre », art. cit., p. 90 , note 60 . 
âmes lors du Jugement Dernier, schéma classique dans la région. Deux chapelles, l'une dans l'église Saint-Barnard de Romans, l'autre dans le prieuré Saint-Pierre de Lémenc en Savoie, révèlent un lien direct avec le monde des morts à cette même période. La première se superpose à « une crypte dédiée à Notre-Dame des Os (des Ossis)» qui «servait de caveau funéraire pour recevoir les ossements provenant des fosses voisines », alors que la seconde est dénommée capella Sancti Michaelis du charnier ${ }^{28}$.

Mais, parmi ces saints guerriers, seul le culte de l'archange est dénué de relique. La documentation de la fin du Moyen Âge, et en priorité les visites pastorales des $\mathrm{XIV}^{\mathrm{e}}$ et $\mathrm{XV}^{\mathrm{e}}$ siècles, mettent en évidence la conservation de nombreux ossements sauf de Michel (figure 4).

\section{Figure 4 - Tableau. Les reliques des saints guerriers ${ }^{29}$}

\begin{tabular}{|c|c|c|}
\hline Bâti ecclésial & Reliques & Date \\
\hline Fontanieu & Saint Maurice & 1488 \\
Saint-Giraud de Varces & Saint Giraud (Géraud) & 1551 \\
Saint-Georges de & Saint Georges & 1455 \\
Commiers & Saint Georges & 1509 \\
Sinard & . & \\
\hline
\end{tabular}

Dans une société où l'usage des reliques apparaît nécessaire afin d'ancrer un lieu de culte au cœur du Moyen Âge, son insertion territoriale et sa diffusion ne sont construites que sur la similarité entre le lieu de fondation d'un autel ainsi que les sites d'apparition et la nécessaire protection d'une communauté.

\footnotetext{
${ }^{28}$ Jules MARIOn, Cartulaire de l'Église cathédrale de Grenoble, op. cit., "Pouillé de 1497 », p. 367-368; Ulysse CheVAlier, La Chapelle de Saint-Michel de Romans, Grenoble, Prudhomme éd., 1869, p. 9.

${ }^{29}$ AD38 (Isère), Procès-verbaux des visites pastorales des évêques de Grenoble, dans les paroisses de Saint-Maurice de Fontanieu par Laurent I Allemand, le 5 juillet 1488, 4 G 261, $\mathrm{f}^{\circ} 134 \mathrm{r}^{\circ}-135 \mathrm{r}^{\circ}$; de Saint-Giraud de Varces par Laurent II Allemand, le 7 avril 1551, 4 G 278, f $\mathrm{f}^{\circ} \mathrm{v}^{\circ}$; et de Saint-Georges de Commiers par Siboud Allemand, le 9 novembre 1455, $4 \mathrm{G}$ 257, $\mathrm{f}^{\circ} 147 \mathrm{v}^{\circ}-149 \mathrm{r}^{\circ}$; et Procès-verbal de la visite pastorale de la paroisse de Sinard par Gaspard de Tournon, évêque de Valence et Die, le 30 septembre 1509, dans Louis FILLET, État des diocèses de Die et de Valence en 1509, Valence, imprimerie Jules Céas et fils, 1882, p. 15.
} 
Pour conclure, répondant au besoin de l'ensemble de la communauté, l'archange semble couronner les sommets orientaux du massif du Vercors dès la fin du haut Moyen Âge. Il se maintient et se présente comme incontournable aux siècles suivants en démontrant une capacité d'adaptation considérable, mais finit par décliner peu à peu. Protecteur de la communauté, intercesseur entre cette dernière, Dieu et le monde des morts, il est encore présent aux $\mathrm{XIV}^{\mathrm{e}}$ et $\mathrm{XV}^{\mathrm{e}}$ siècles où il répond à une exigence plus individuelle: l'effacement des sites michaéliques initiaux des $\mathrm{X}^{\mathrm{e}}-\mathrm{XI}^{\mathrm{e}}$ siècles se fait au profit de nouveaux lieux de culte à l'archange dans une religiosité renouvelée.

L'essoufflement est notable au bas Moyen Âge. Lorsque l'ecclesia de Eclesiolis disparaît au profit d'un prieuré, sa chapelle reprend le patronage à Michel auquel on adjoint saint Blaise, très présent dans ces vallées, et cette association vise à créer une nouvelle dynamique. Cependant, à Uriol, l'arrivée d'un culte à saint Loup, associé à celui de l'archange entre 1473 et 1488 , met fin à la dédicace initiale ${ }^{30}$.

Enfin, ce déclin du culte michaélien peut être mis en relation avec celui des reliques, tous deux répondant à un besoin de protection. Les archives de la fin du $\mathrm{XV}^{\mathrm{e}}$ et du début du $\mathrm{XVI}^{\mathrm{e}}$ siècle dévoilent leur présence en nombre dans les églises des paroisses de ce territoire. Elles ont été amenées lors de la consécration initiale, ou «ajoutées 》 a posteriori afin de renforcer la sacralité de l'autel, mais peu sont authentifiées ${ }^{31}$. Au fil du temps, le désintérêt que leur portent les populations est notable : comme les titulatures premières à l'archange, elles ne sont plus nécessaires pour légitimer l'existence de l'église.

\footnotetext{
${ }^{30}$ AD38 (Isère), Procès-verbaux des visites pastorales de la paroisse d'Uriol par Siboud Allemand, évêque de Grenoble, le 11 juin 1473, 4 G 260, fo $50 \mathrm{r}^{\circ}$; et par Laurent I Allemand, évêque de Grenoble, le 5 juin 1488, 4 G 261, fo $135 \mathrm{v}^{\circ}-136 \mathrm{r}^{\circ}$.

${ }^{31}$ Merci à Clémence Revest pour ses réflexions sur la perte du sens premier des reliques dans un lieu de culte.
} 